\title{
Real time weather surveillance via lab view interfaced arduino
}

\author{
Abhishek Anil Mungekar ${ }^{1}$, Yashraj Kalpesh Solanki ${ }^{1}$, R. Swarnalatha ${ }^{2}$ * \\ ${ }^{1}$ Dept of Electronics and Instrumentation Engineering, Birla Institute of Technology \& Science, Pilani, Dubai Campus, Dubai. U.A.E \\ ${ }^{2}$ Assistant Professor, Dept of EEE,Birla Institute of Technology \& Science, Pilani, Dubai Campus, Dubai. U.A.E \\ *Corresponding author E-mail: swarnalatha@dubai.bits-pilani.ac.in
}

\begin{abstract}
Background: In modern day scenario, especially in the United Arab Emirates, many fluctuations can be seen in the climatic conditions. These type of climate changes can adversely affect the workplace environment of several industries, their transport lines, and as well as day to day functioning of various other human activities.

Approach: The idea proposed, is an all-inclusive metrological real time weather monitoring system using the advanced Bosch barometric pressure sensor BMP180 and Adafruit humidity sensor DHT22. The monitoring is achieved by making use of efficient virtual simulation (LabVIEW) which is directly interfaced with ATmega328 quartz crystal $16 \mathrm{MHZ}$ microprocessor.

Results: From the real time LabVIEW display, several weather parameters are monitored, in an attempt to maintain them below a certain required threshold. Conclusions: The results suggest a setup that makes the entire monitoring process cost effective and easy to operate due to its user friendly interface.
\end{abstract}

Keywords: Bmp180; DHT22; LabVIEW; Atmega328.

\section{Introduction}

In this contemporary world, precision and accuracy has become of the utmost importance in the field of metrology [2]. Acquiring necessary weather parameters meticulously, can help functioning of the transportation lines, industries, factories etc. efficiently. But, it is known for a fact that, as precision increases the cost of obtaining information also increases, hence reducing the accessibility.

Every industry needs to adhere to certain norms set up by the government, to ensure an apt workplace for the laborers [3]. Hence, obtaining specific data regarding the workplace such as ; temperature, humidity and several other parameters is very important in this hot and humid country [4] [5] [8].

It isn't possible for every industry to monitor such parameters precisely, especially for the smaller and less established factories [6] [7], as installing such data acquiring systems could cost them a fortune.

The paper elucidates as to how these certain parameters can be monitored in the most precise and cost effective way, making it easily accessible. For this, an advanced humidity sensor, DHT22 is made use of to give accurate data on the humidity of the surrounding environment [10]. BMP180 is used to give accurate barometric pressure readings and temperature readings [11], and finally a Light dependent resistor (LDR) to give precise light intensity readings of the surrounding environment.

\section{Methods and materials required}

The prior requirement of the project is to interface LabVIEW with Arduino [9], this is accomplished by downloading VI package manager [1] and by using a simplified string to array converter. These two methods have proven to be cost effective and highly efficient. To add to further simplification, digital sensors such as barometric pressure and humidity sensors have been made use of. The following materials are used for this purpose: -

1) Arduino Uno R3 Board $x 3$

2) LabVIEW Software

3) DHT-22 (Digital Humidity Transducer).x1

4) BMP180 (Barometric pressure sensor) $\mathrm{x} 1$

5) LDR (Light Dependent Resistor)............. 1

For interfacing the two soft wares, two methods are mainly used the first one pertaining to analog sensors (LDR) while the second method is more appropriate for digital sensors (BMP180 and DHT22).

\subsection{Method 1 for generalized analog sensors}

The principle of LDR is that, its sensitivity varies with respect to the light wavelength. The key requirement for this is the VIPM (virtual instrumentation package manager) which directly projects the Arduino's analog to digital converted parameters. The reason to choose this method is because the VIPM handles all of the required installations in the subsequent directories where LabVIEW is present and the respective version of LabVIEW is used automatically. There is no Arduino coding required with respect to the LDR sensor used. This is where error detection and troubleshooting becomes easy. The interface is loaded onto the Arduino (LIFA base) for completing the interfacing procedure.

Note: download the NI-VISA driver which enables the computer to detect any hardware connections to it.

The following is a generalized block diagram for the LDR constructed in LabVIEW using the Arduino inputs. For better understanding real time indicters and graphical representation have been made use of. The input provided by the Arduino (Arduino read pin in the diagram) runs at a baud rate of 115200 symbols per second. Comparators are used to further simplify the indication process. 


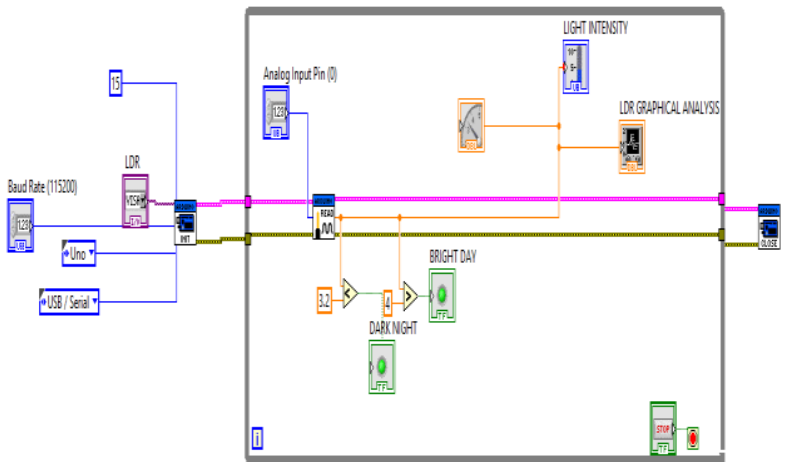

Fig. 1: Generalized Block Diagram of Light Dependent Resistor.

\subsection{Method 2- for generalized digital sensors}

BMP180 has an in built ADC which provides digital PWM signals. A major advantage of using this is the provision of indemnified barometric pressure and temperature parameters. This method utilizes simplified, string to array converter blocks capturing data with respect to the offset provided in a sequential order. The following is the block diagram for the BMP180: -

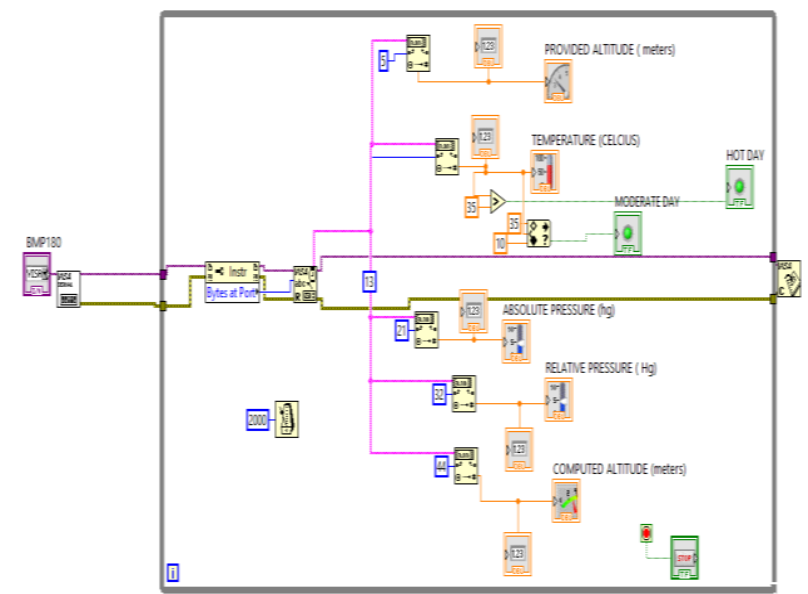

Fig. 2: Generalized block diagram of BMP180 barometric sensor.

Initially the Arduino program is uploaded. The VISA resource block obtains the uploaded values from the respective COM port (depends on the connectivity of the Arduino board) onto the VI of LabVIEW .Since BMP180 provides five outputs namely Absolute pressure, Relative pressure, Composed Altitude, Provided altitude and temperature. It is essential in order to differentiate these variable outputs received at a single COM port. For this crucial purpose the "Bytes at Port" block is made use of. Corresponding to the "Bytes at Port" block, are the vital string to fractional array converters which help in projecting the corresponding output parameters on the front panel with respect to the induced offsets (variable based on the Arduino program written which can be altered to meet the changing requirements accordingly). A time delay is introduced to give regular output readings. This time delay accounts for any fluctuations in the measured parameters. DHT22 utilizes polymer capacitive sensing element hence providing it an operating range of 0 to 100 percent of relative humidity and -40 degree to 80 degrees Celsius of temperature.

The following is a block diagram of DHT22: -

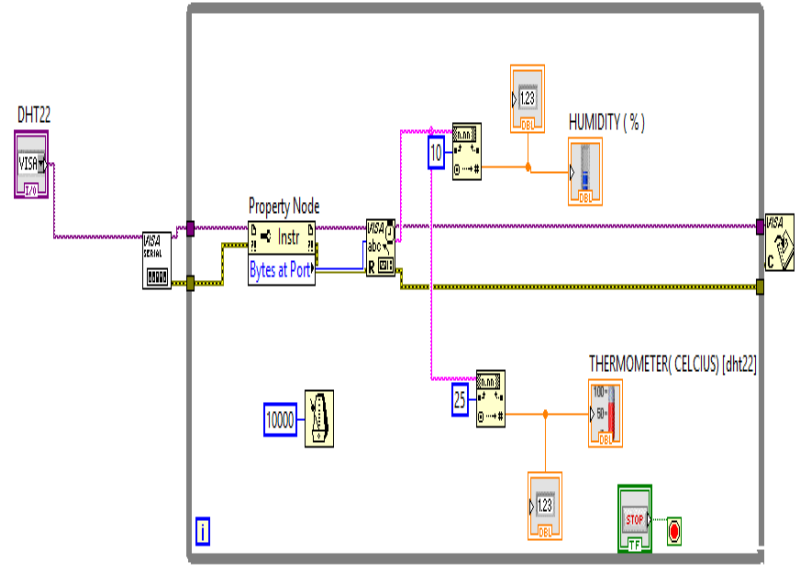

Fig. 3: Generalized Block Diagram of DHT22 Humidity Sensor.

The program for DHT22 is first uploaded on Arduino; the output is captured by the VISA resource and flows to the "Bytes at Port" block through the VISA serial block. On providing certain offset, the values of temperature and humidity can be detected separately via the string to fractional array converters. A time delay of 10 seconds is introduced in order to regularly record and observe the parameters, accordingly taking corrective actions.

\section{Results}

With the block diagrams obtained for respective sensors, the panels for light intensity, temperature, humidity, absolute pressure, relative pressure, composed altitude, relative altitude are shown in Fig 4, 5,6 .

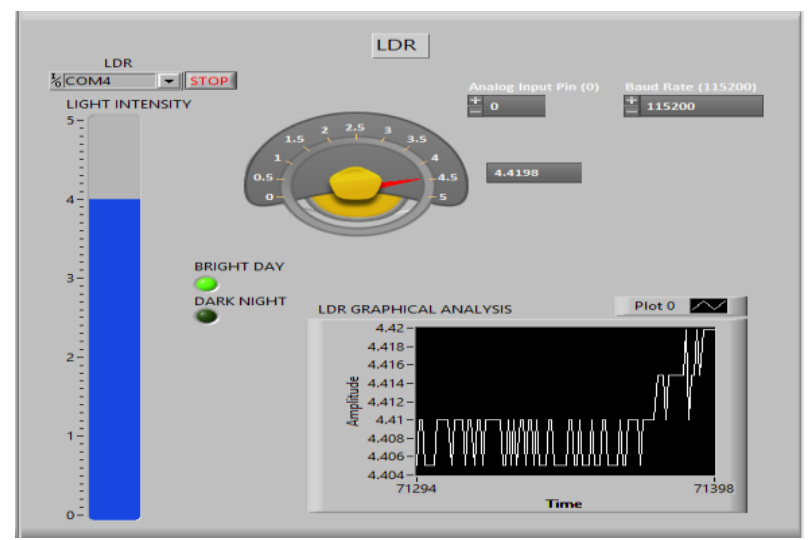

Fig. 4: Front Panel View of the Light Dependent Resistor Sensor Readings.

The above figure (Fig 4) an elaborate analytical panel, giving detailed information on the variation of light intensity in the surrounding environment.

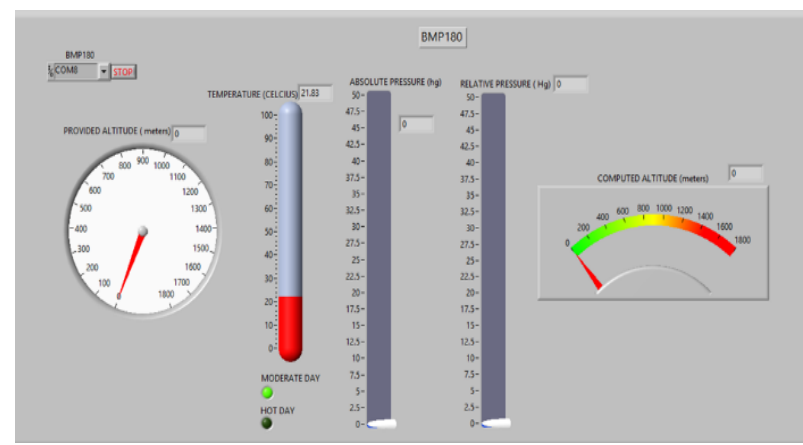

Fig. 5: Front Panel View of the Barometric Pressure Sensor BMP180. 
The above illustrated panel (Fig 5), projects sequential readings on temperature, pressure, and the altitude (Every reading obtained after $2 \mathrm{sec}$ of delay simultaneously).

The below shown panel (Fig 6) gives a descriptive analysis of the humidity and also temperature (temperature is also calculated by BMP180, both these readings can be used as a cross reference to each other).

The measurements are taken at an interval of every $10 \mathrm{sec}$ (variable from user to user).The interval is taken such that the activities of the BMP180 are synchronized with the humidity and temperature readings of the DHT22.

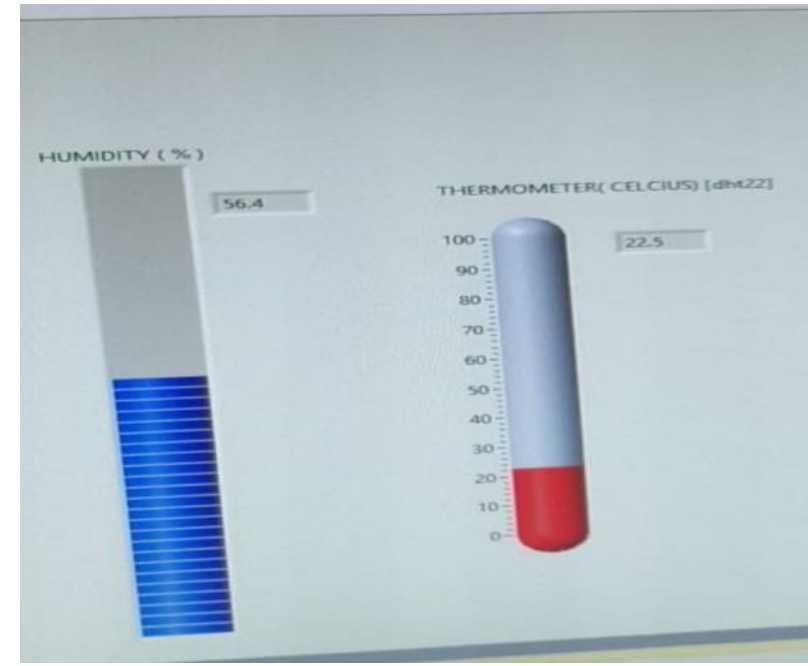

Fig. 6: Front Panel View of Humidity Sensor DHT22.

\section{Conclusion}

The paper makes use of advanced techinqies to measure surrounding envionemnt parameters and give the metrological analysis in real time. The panel set up is used to regularly monitor these parameters and thus help in taking further actions in order to mainatin these parameters. Hence, ensuring an appropriate workspace enviornemnt.

\section{Acknowledgements}

The authors would sincerely like to thank Prof. R. N. Saha, Director, BITS Pilani, Dubai Campus for his constant encouragement and support.

\section{References}

[1] Francisco Javier Jimenez ; Francisco Ramon Lara ; Maria Dolores Redel , API for communication between Labview and Arduino UNO , Browse Journals \& Magazines, IEEE Latin America Transaction, Volume: 12 Issue: 6 .

[2] Robert D.Larrabee , Michael T.Postek , "Precision, accuracy, uncertainty and traceability and their application to submicrometer dimensional metrology". Solid-State Electronics Volume 36, Issue 5, May 1993, Pages 673-684 https://doi.org/10.1016/00381101(93)90234-H.

[3] Zayed Bin Sultan Al Nahyan, President of the United Arab Emirates , UAE Labour Law, section 11 , Issued at the Presidential Palace in Abu Dhabi On the 6th of Jumada Al Aakhir, $1400 \mathrm{H}$ Corresponding to the 20th of April $1980 \mathrm{G}$.

[4] WMO , - , 2008: Guide to Meteorological Instruments and Methods of Observation (WMO-No. 8). Geneva.

[5] WMO, 2010: Guide to the Global Observing System (WMO-No. 488). Geneva

[6] Anthony C. Mulligan, Issues for Small Manufacturing Enterprises New Directions in Manufacturing: Report of a Workshop (2004).

[7] Connecting Small Manufacturers with the Capital Needed to Grow, Compete, and Succeed:
[8] Small Manufacturers Capital Access Inventory and Needs Assessment Report , November 2011.

[9] Chan, C.B.; Ryan, D.A., Assessing the Effects of Weather Conditions on Physical Activity Participation Using Objective Measures. Int. J. Environ. Res. Public Health 2009, 6, 2639-2654. https://doi.org/10.3390/ijerph6102639.

[10] Schwartz, M., Programming Arduino with LabVIEW, Published by Packt Publishing Ltd., ISBN 978-1-84969-822-1, (2015).

[11] Monk, S., 30 Arduino Projects for the Evil Genius, the McGrawHill Companies, ISBN: 978-0-07-174134-7, (2010).

[12] HAN Zhi-jun, LIU Xin-min.DS18B20 digital temperature sensor and its application. Nanjing Institute of Technology.2003 (03):9-12. 